\title{
Understanding the Metabolic Fate and Bioactivity of Dietary Anthocyanins ${ }^{\dagger}$
}

\author{
Gabriele Netzel 1,*, Olivia Wright ${ }^{2}$, Yasmina Sultanbawa ${ }^{1}$, Michael Netzel ${ }^{1}$ \\ 1 ARC Industrial Transformation Training Centre for Uniquely Australian Foods, Queensland Alliance for \\ Agriculture and Food Innovation, The University of Queensland, Coopers Plains, Australia; \\ y.sultanbawa@uq.edu.au (Y.S.); m.netzel@uq.edu.au (M.N.) \\ 2 School of Human Movement and Nutrition Sciences, The University of Queensland, St Lucia, Australia; \\ o.wright@uq.edu.au \\ * Correspondence: g.netzel@uq.edu.au \\ + Presented at the third International Tropical Agriculture Conference (TROPAG 2019), Brisbane, Australia, \\ 11-13 November 2019.
}

Published: 19 January 2020

\begin{abstract}
Anthocyanins are plant pigments and dietary phytochemicals, and may have potential health benefits. There is emerging evidence from epidemiological and experimental studies that suggests a higher consumption of anthocyanin-rich foods is associated with a reduced risk of heart disease and diabetes. To better understand the observed beneficial effects of anthocyanins and their underlying mode of action, bioavailability and metabolic fate needs to be studied in more detail. Healthy human subjects (10-12 in two different studies) received red grape pomace (700 $\mathrm{mg}$ anthocyanins/mainly as malvidin-3-glucoside) or Queen Garnet plum (QGP) juice (426 mg anthocyanins/mainly as cyanidin-3-glucoside) and an anthocyanin-free control in a randomised crossover design. Malvidin- and cyanidin-glycosides are common in many fruits and beverages such as red grapes, red grape juice, red wine, blueberry, cherry, elderberry, (Japanese) plum and are therefore of dietary significance. 24-hr urine samples were collected and analysed for anthocyanins and metabolites by UHPLC-PDA-MS. Methylated, glucuronidated and sulphated anthocyanins could be identified as characteristic metabolites in both studies. Furthermore, the increase in urinary hippuric acid (microbial/hepatic metabolite) was considerable in both studies after the consumption of red grape pomace or QGP juice (1.8-4.5-fold vs. control; $p<0.05)$. These findings suggest that structurally different anthocyanins are exposed to a similar extensive metabolism by enzymes and the gut microbiome and that the generated metabolites are most likely the bioactive compounds in vivo. Therefore, more human studies are warranted to investigate the metabolic fate of dietary anthocyanins and the bioactivity of generated metabolites.
\end{abstract}

Keywords: anthocyanins; bioavailability; metabolism; urine; humans

Note: Ethics code 1567-07/05 (Friedrich-Schiller University Jena, Jena, Germany); Red Grape Pomace study; Ethics code 2012000182 (The University of Queensland, St. Lucia, QLD, Australia); Queen Garnet Plum Juice study.

Funding: This research was partly funded by HAL, SF10012.

Conflicts of Interest: The authors declare no conflict of interest.

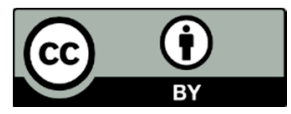

(C) 2020 by the authors. Licensee MDPI, Basel, Switzerland. This article is an open access article distributed under the terms and conditions of the Creative Commons Attribution (CC BY) license (http://creativecommons.org/licenses/by/4.0/). 